\title{
Diffractive Deep-Inelastic Scattering with a Leading Proton at HERA-2
}

\author{
Mikhail Kapishin (for the H1 Collaboration) \\ Joint Institute for Nuclear Research, Joliot Curie 6, 141980 Dubna, Russia, kapishin@mail.desy.de
}

\begin{abstract}
Results are reported on a new measurement of the cross section for diffractive deepinelastic scattering process $e p \rightarrow e X p$ with the leading final state proton detected in the H1 Forward Proton Spectrometer, using data collected at HERA-2. The data are compared to QCD predictions at next-to-leading order based on diffractive parton distribution functions previously extracted from measurements of inclusive diffractive deep-inelastic scattering with a large gap in the rapidity distribution of the final state hadrons.
\end{abstract}

\section{Introduction}

Diffractive processes such as $e p \rightarrow e X p$ have been studied extensively in deep-inelastic electron-proton scattering (DIS) at the HERA collider [1, 2, 3, 4]. Their understanding at fundamental level is crucial for the development of quantum chromodynamics (QCD) at high parton densities.

The photon virtuality $Q^{2}$ provides a hard scale for perturbative QCD to be applicable, so that diffractive DIS events can be viewed as processes in which the photon probes a net colour singlet combination of exchanged partons. A hard scattering QCD collinear factorisation theorem [5] allows 'diffractive parton distribution functions' (DPDFs) to be defined, which describe the partonic structure of that exchange.

In a number of previous analyses, including [1], diffractive DIS events were selected on the basis of the presence of a large rapidity gap (LRG) between the leading proton and the remainder of the hadronic final state $X$. A complementary way to study diffractive processes is by direct measurement of the outgoing proton using Forward Proton Spectrometers (FPS) $[2,3,4]$. The FPS method of studying diffraction has several advantages over the LRG method, although the FPS acceptance is low. In contrast to the LRG case, the squared fourmomentum transfer at the proton vertex $t$

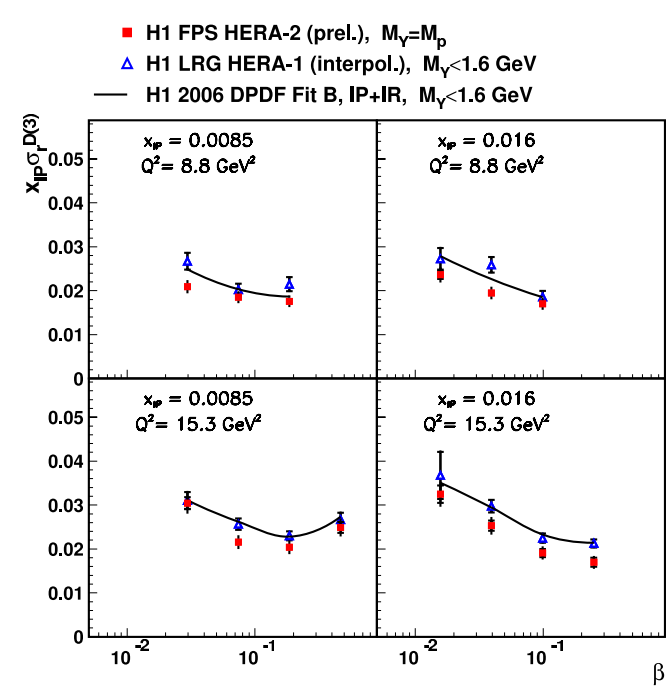

Figure 1: The diffractive reduced cross section, shown as a function of $\beta$ for selected values of $x_{\mathbb{P}}$ and $Q^{2}$. The H1 FPS data are compared to the H1 LRG results. The solid curves represent the results of the 'H1 2006 DPDF Fit B' to the LRG data. 
can be reconstructed. The FPS method also selects events in which the proton scatters elastically, whereas the LRG method does not distinguish the elastic case from dissociation to excited systems $Y$ with small masses $M_{Y}$. The FPS also allows measurements up to higher values of the fractional proton longitudinal momentum loss $x_{\mathbb{P}}$ than is possible with the LRG method, extending into regions where the sub-leading trajectory is the dominant exchange. Together, the FPS and LRG data thus provide a means of testing in detail whether the variables $x_{\mathbb{P}}, t$ and $M_{Y}$ associated with the proton vertex can be factorised from the the variables $\beta=x / x_{\mathbb{P}}$ and $Q^{2}$ describing the hard interaction with the photon. Here $\beta$ is the longitudinal momentum fraction of the colour singlet carried by the struck quark, $x$ is the Bjorken scaling variable.

\section{Results}

In this report, a new measurement of the reduced cross section $\sigma_{r}^{D}$ for the diffractive DIS process $e p \rightarrow e X p$ is presented, using the FPS data collected with the H1 detector at HERA2. $\sigma_{r}^{D}$ is related to the diffractive structure functions $F_{2}^{D}$ and $F_{L}^{D}$ by:

$$
\sigma_{r}^{D}=F_{2}^{D}-\frac{y^{2}}{1+(1-y)^{2}} F_{L}^{D}
$$

where $y$ is the inelasticity. Thus $\sigma_{r}^{D} \simeq F_{2}^{D}$ is a good approximation except at the highest $y$. The analysed data sample corresponds to an integrated luminosity of $156 \mathrm{pb}^{-1}$. The data cover the range $x_{\mathbb{P}}<0.1,4<$ $Q^{2}<700 \mathrm{GeV}^{2}$ and $0.001<$ $\beta<1$. The statistics of DIS events with a leading proton are 20 times higher than those collected with the H1 FPS at HERA-1 [2]. The FPS HERA2 measurements extend the kinematic range to higher $Q^{2}$. The diffractive reduced cross section $\sigma_{r}^{D(3)}$ is measured differentially in $x_{\mathbb{P}}, \beta$ and $Q^{2}$. The data are integrated over the range $|t|<$ $1 \mathrm{GeV}^{2}$, which is the region covered by H1 using the LRG method [1]. The measurement of $x_{\mathbb{P}} \sigma_{r}^{D(3)}$ is presented in Figure 1 as a function of $\beta$ for selected values of $x_{\mathbb{P}}$ and $Q^{2}$. The data are compared to the H1 LRG data interpolated to the FPS $\beta, Q^{2}$

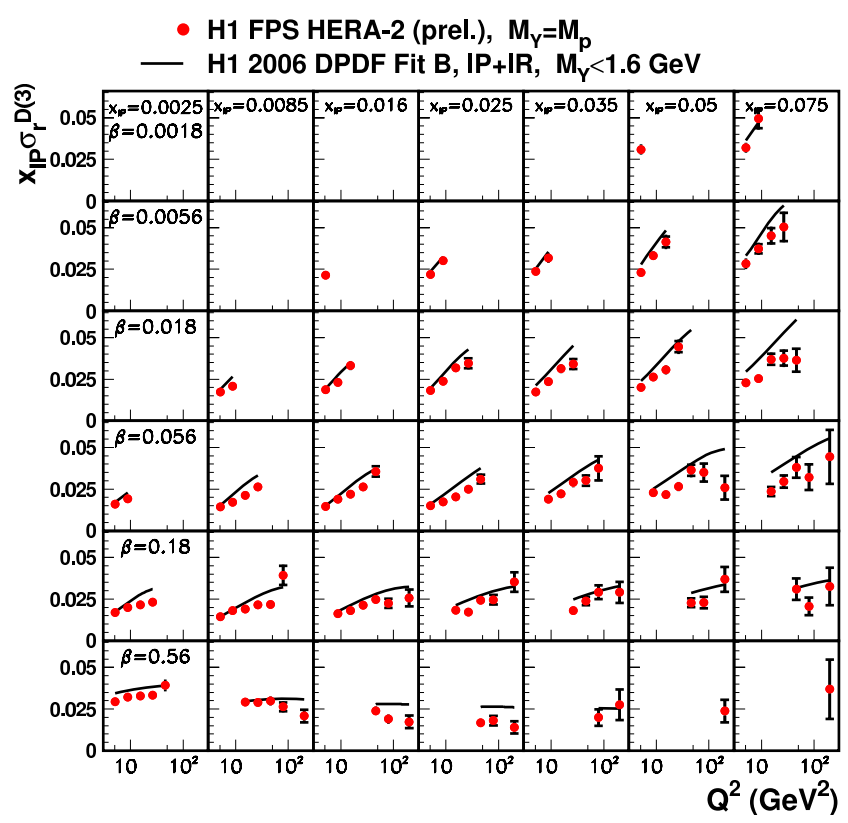

Figure 2: The diffractive reduced cross section, shown as a function of $Q^{2}$ for different values of $x_{\mathbb{P}}$ and $\beta$. The solid curves represent the results of the 'H1 2006 DPDF Fit B' to the LRG data. 
and $x_{\mathbb{P}}$ values. Predictions derived from the 'H1 2006 DPDF Fit B' [1] performed to the H1 LRG data in the framework of next-to-leading order (NLO) DGLAP evolution are also shown. The statistical precision of the FPS measurement reached that for the published H1 LRG data. The difference in the normalisation is attributed to the proton dissociation contribution to the LRG sample. The FPS HERA-2 data are measured with an averaged systematic uncertainty of $8 \%$ and a normalisation uncertainty of $6 \% \cdot \sigma_{r}^{D(3)}$ is decreasing with $\beta$ over most of the measured kinematic range, but rises at high $\beta$ and low $Q^{2}$ and $x_{\mathbb{P}}$. Within the framework of DPDFs, this can be explained by the shape of diffractive quark densities [1].

The $Q^{2}$ dependence of $\sigma_{r}^{D(3)}$ at fixed $x_{\mathbb{P}}$ and $\beta$ (Figure 2) is characterised by positive scaling violations $\left(\partial \sigma_{r}^{D(3)} / \partial \ln Q^{2}>0\right)$ throughout the kinematic range, except at the highest $\beta$. This observation is consistent with that from H1 measurements using the LRG method and implies a large gluonic component in the DPDFs.

In Figure 3 the FPS $\sigma_{r}^{D(3)}$ results are compared to those of the ZEUS collaboration, measured using their Leading Proton Spectrometer (LPS) [4] and also integrated over $|t|<1 \mathrm{GeV}^{2}$. The ZEUS data points are interpolated to the $\beta, Q^{2}$ and $x_{\mathbb{P}}$ values of this measurement using a parameterisation of the 'H1 2006 DPDF Fit B'. Within the normalisation errors of $6 \%$ for H1 FPS data and $(+11 \% /-7 \%)$ for the ZEUS LPS data, there is an agreement between the two data sets. The H1 FPS measurements extend the kinematic range to higher $Q^{2}$.

The FPS data are also compared directly to the LRG measurement in order to test the compatibility between the results obtained with the two measurement techniques and to quantify the proton dissociation contribution to the LRG data. The diffractive cross section measured with the LRG data is defined to include proton dissociation to any system $Y$ with a mass in the range $M_{Y}<1.6 \mathrm{GeV}$, whereas $Y$ is defined to be a proton in the cross section measured with the FPS. The LRG results are interpolated to the $Q^{2}, \beta$ and $x_{\mathbb{P}}$ bin center values of the FPS data using a parameterisation of the 'H1 2006 DPDF Fit B'. Since the two data sets are statistically independent and the dominant sources of systematic error are very different, correlations between the uncertainties on the FPS and LRG data are neglected.

The ratio of the LRG to the FPS cross section is plotted in Figure 4 as a function of $Q^{2}$, $\beta$ and $x_{\mathbb{P}}$, after averaging over the other kinematic variables. The combined normalisation 
error is $8.5 \%$. Within the remaining uncertainties of typically $6 \%$ per data point. No significant dependence of the ratio on $\beta, Q^{2}$ or $x_{\mathbb{P}}$ is observed.

The ratio of overall normalisations, LRG / FPS, is $\sigma\left(M_{Y}<1.6 \mathrm{GeV}\right) / \sigma(Y=p)=$ $1.18 \pm 0.01$ (stat.) \pm 0.06 (syst.) \pm 0.10 (norm.) the dominant uncertainties arising from the normalisations of the FPS and LRG data. This result is in agreement within the uncertainties with the value of $1.23 \pm 0.03$ (stat.) \pm 0.16 (syst.) obtained from comparison of the H1 LRG and FPS data at HERA-1 [2].
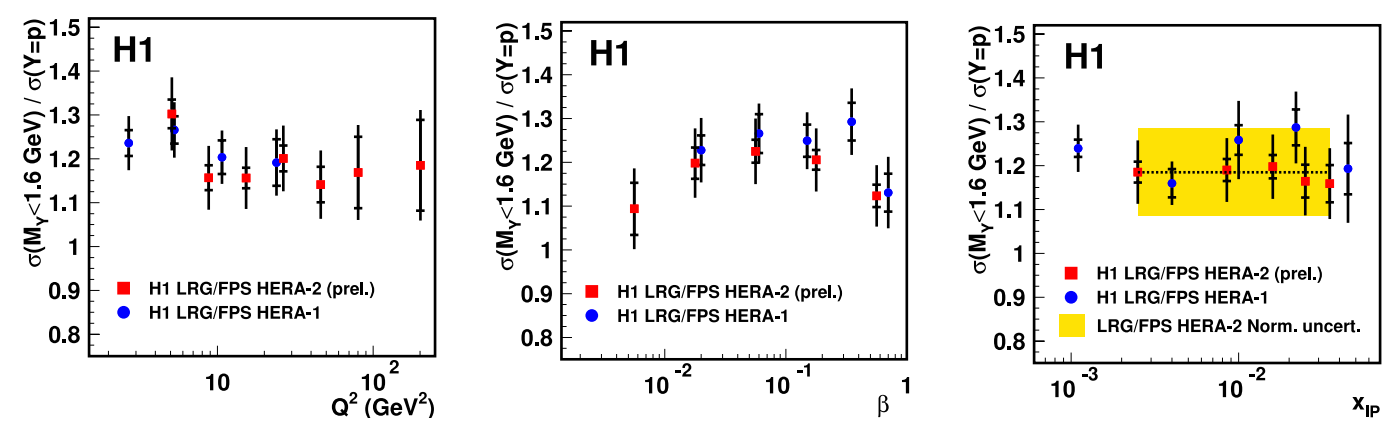

Figure 4: The ratio of the diffractive cross section for $M_{Y}<1.6 \mathrm{GeV}$ to that for $Y=p$ obtained from $\sigma_{r}^{D(3)}$ measurements using the H1 LRG data [1] and the FPS data at HERA1 [2] and HERA-2. The results are shown as a function of $Q^{2}, \beta$ and $x_{\mathbb{P}}$, after averaging over two other variables. Normalisation uncertainty of $8.5 \%$ is shown. The dash line represents the result of a fit to the data assuming no dependence on $x_{\mathbb{P}}$.

The good agreement, after accounting for proton dissociation, between the LRG and the FPS data confirms that the two measurement methods lead to compatible results, despite having very different systematics. The lack of a strong kinematic dependence of the ratio of the two cross sections shows, within uncertainties, that proton dissociation with $M_{Y}<$ 1.6 GeV can be treated similarly to the elastic proton case. This supports the factorisation in terms of $M_{Y}$, for both the pomeron and the sub-leading exchange contributions, of processes occurring at the proton vertex from those describing the hard interaction. It also confirms that contributions from proton dissociation in the LRG measurement do not significantly alter the measured $\beta, Q^{2}$ or $x_{\mathbb{P}}$ dependences and hence cannot have a large influence on the diffractive gluon density or other information extracted from the LRG data.

\section{References}

[1] H1 Collaboration, Eur. Phys. J. C 48 (2006) 715.

[2] H1 Collaboration, Eur. Phys. J. C 48 (2006) 749.

[3] ZEUS Collaboration, Eur. Phys. J. C 38 (2004) 43.

[4] ZEUS Collaboration, Nucl. Phys. B 816 (2009) 1.

[5] J. Collins, Phys. Rev. D 57 (1998) 3051 [Erratum-ibid. D 61 (2000) 019902]. 